\title{
Satisfacción laboral y compromiso organizacional en empleados de una universidad privada de Lima
}

\author{
Carlos Francesco Marquina-Vergara* \\ Instituto de Idiomas, Universidad Peruana Unión, Alt. Km 19; Carretera Central, Chosica, Lima
}

\begin{abstract}
RESUMEN
La satisfacción laboral y el compromiso organizacional son dos constructos que presentan un gran interés para los psicólogos y en especial para aquellos dedicados al área de Recursos Humanos. Por tal motivo, la presente investigación indagó ambos constructos y su relación en los empleados de una universidad privada en el año 2011. Se aplicó la Escala de Satisfacción Laboral SL - SPC (Palma, 1999) y la Escala del Grado de Compromiso de los Empleados Universitarios ASD (Grajales, 2000) a 121 personas pertenecientes a una población de 300 individuos en condición de empleados. Los resultados revelan que existe insatisfacción laboral en un 51.3\%, siendo las dimensiones condiciones laborales/materiales y políticas administrativas con las cuales los empleados se sienten más insatisfechos; sin embargo, pese al resultado anterior, el 91\% presenta altos niveles de compromiso organizacional, por lo que se sienten identificados con la institución y le brindan su lealtad. La correlación de Pearson para las variables de estudio de 0.73 , el cual es estadísticamente significativa $(\mathrm{p}=0.10)$ en la población estudiada.
\end{abstract}

Palabras clave: Satisfacción laboral, compromiso organizacional, psicología organizacional/industrial, recursos humanos.

\begin{abstract}
Job satisfaction and organizational commitment are two constructs that are of great interest to psychologists and especially for those dedicated to Human Resources. Therefore, this research has investigated both constructs and its correlation in private university employees in 2011. We applied the Job Satisfaction Scale SL - SPC (Palma, 1999) and the Scale of Grade of SDA University Employee Commitment (Grajales, 2000) to 121 people belonging to a total population of 300 individuals who had the employee status. The results reveal that, there is Job dissatisfaction in a 51.3\%, where the dimensions: Working Conditions / Materials and Management Policy are issues that causes mayor dissatisfaction among employees; however, and despite of the previous result, the $91.2 \%$ have high levels of organizational commitment, for this reason, they feel identify with and give their loyalty to the Institution. The Pearson correlation between our two variables was 0.73 , which is statistically significant $(p=0.10)$ in the studied population.
\end{abstract}

Keywords: Job satisfaction, organizational commitment, organizational / industrial psychology, human resources.

*Correspondencia: francesco.carlos@hotmail.com,51-1-989266931 


\section{INTRODUCCIÓN}

Las investigaciónes sobre la satisfacción laboral y el compromiso organizacional no deberían ser catalogadas como tareas anuales, rutinarias y sistemáticas que requieran las instituciones, ya que, a raíz de su estudio, pueden crearse o fortalecerse políticas institucionales para mejorar las condiciones laborales en las organizaciones y aumentar el desempeño laboral de sus integrantes.

Se ha percibido que en la última década las organizaciones han reconocido y aceptado que el capital más importante son las personas que las conforman. Es por esta razón que se incrementaron considerablemente las investigaciones basadas en determinar cómo atraer, mantener, incentivar y retener a los mejores colaboradores, a fin de que sigan brindando sus fuerzas en beneficio de la institución a las cuales representan.

En el transcurso del tiempo, diversos autores han tratado de definir estas variables (la satisfacción laboral y el compromiso organizacional) intentando comprenderlas cabalmente para aplicarlas a la realidad laboral de las organizaciones; como fruto de su estudio metódico hoy entendemos y aceptamos la satisfacción laboral como la actitud del trabajador hacia su propio trabajo (Palma, 1999) y al compromiso organizacional como la fuerza relativa de identificación $\mathrm{y}$ de involucramiento de un individuo con una organización en particular (Steers 1991).

$\mathrm{Al}$ evaluar las investigaciones sobre la satisfacción laboral encontramos que los trabajadores con altos niveles de satisfacción laboral tienen un sentimiento positivo en relación a su actividad laboral y hacia la institución a la cual pertenecen, sin embargo, aquellos trabajadores que poseen bajos niveles de satisfacción o sea que se encuentran insatisfechos tendrán sentimientos negativos y presentarán diferentes tipos de comportamientos no productivos y que resultarán muy perjudiciales para las empresas (Côté y Heslin, 2003).

Por otro lado, el compromiso organizacional ha sido muy estudiado en los sectores públicos, privados y sin fines de lucro y más recientemente de manera internacional.

Las primeras investigaciones se centraban en definir el concepto mientras que las actuales continúan examinando el compromiso organizacional a través de dos corrientes de estudio populares, (1) las actitudes relacionadas al compromiso y (2) los comportamientos relacionados al compromiso; estas dos corrientes han constituido el hilo guiador para el estudio de este constructo a través de los años.
Este avance, por lo tanto, fue productivo y significativo; como prueba palpable tenemos una gran variedad de antecedentes los cuales han sido identificados y aceptados durante los pasados 30 años (Hall, 1968).

Debemos recalcar que el compromiso organizacional es un tema extremadamente importante que toda organización debería entender. El nivel en el cual un empleado está relacionado con su trabajo (involucramiento laboral), comisionado y creyente en las metas organizacionales y sus propósitos (compromiso organizacional), deseos de trabajar (ética laboral), y su comisión hacia una carrera específica o profesión pueden tener un impacto en la organización en general. En la economía de hoy, donde las organizaciones están a la expectativa de producir más con menos recursos, es extremadamente importante el mantener a sus empleados altamente satisfechos y comprometidos.

A manera de resumen mencionamos que la satisfacción laboral y el compromiso laboral son dos variables que influyen fuertemente en el ámbito laboral. El conocerlas y estudiarlas correctamente acarrearía más beneficios que pérdidas; sabemos que realizar estas evaluaciones tiene un costo operativo, sin embargo, los resultados obtenidos serán armas poderosas para realizar una correcta planificación estratégica centrada en aumentar la productividad, en primer lugar, de los trabajadores y por ende de la institución en donde laboran.

\section{MATERIAL Y MÉTODOS}

Esta investigación es de tipo básico porque a través de este estudio se puede ampliar el conocimiento de la satisfacción y el compromiso organizacional (Bunge 1979); además es de tipo cuantitativo porque está fundamentada en base a análisis estadísticos (Hernández, Fernández y Baptista, 2010).

Asimismo, esta investigación es de diseño no experimental, porque no se realiza manipulación de las variables; es transversal debido a que es aplicado en un momento específico en el tiempo; es descriptiva debido a que se evidencian los comportamientos de las variables con el objeto de estudio, y por último es correlacional porque se pretende demostrar la relación existente entre las variables (Hernández, et al. 2010).

La población de estudio está conformada por los trabajadores que tienen un contrato laboral a tiempo indefinido (300 empleados) en esta universidad privada 
de Lima, los cuales laboran en alguna de estas áreas: administrativa, académica y operaria.

Para conocer la cantidad de participantes del estudio, se estimó la muestra probabilística para una población finita utilizando la fórmula del muestreo simple de Sierra-Bravo, cuyo resultado obtenido a un $95 \%$ de confiabilidad fue 169 empleados; a este valor resultante se le aplicó el factor de corrección estadística, el cual reformuló la cantidad de la muestra en un total de 108 empleados.

Luego se procedió a recolectar la data utilizando la nómina de empleados, la cual fue brindada por Recursos Humanos de esta institución. Se entregó las escalas a todas aquellas personas dentro de la nómina que voluntariamente aceptaban ser partícipes del estudio hasta alcanzar la muestra de 108; sin embargo, se logró obtener la participación voluntaria de 121 empleados.

En relación a los instrumentos, para medir la satisfacción laboral, se utilizó la Escala de Satisfacción Laboral SL-SPC de Sonia Palma (1999), la cual fue validada en una muestra de 952 trabajadores de Lima Metropolitana. Los resultados fueron analizados en todos los casos con un nivel de confiabilidad adecuado para la escala global $(\mathrm{a}=0.68)$.

Del mismo modo, el compromiso organizacional fue evaluado con la Escala del Grado de Compromiso de los empleados universitarios adventistas del séptimo día de Tevni Grajales (2001), fue validada en una muestra de 456 administrativos, profesores y empleados de oficina relacionados con el área académica de universidades adventistas del séptimo día de Latinoamérica y España. Los resultados fueron analizados, en todos los casos, con un nivel de confiabilidad adecuado para la escala global $(a=0.87)$. Sin embargo, esta prueba estaba parcialmente creada y validada en el Perú y en otros países hispanos, por lo que, por motivos de confiabilidad de nuestro estudio, se decidió realizar un nuevo análisis a los datos obtenidos luego de su aplicación. El coeficiente de confiabilidad o alfa de Cronbach resultante fue de $\mathrm{a}=0.70$, haciendo que el instrumento sea confiable para nuestro entorno.

El análisis de datos se realizó por medio de estadísticos descriptivos e inferenciales; los cálculos fueron realizados por medio del Paquete Estadístico para las Ciencias Sociales (SPSS vol.21.0); luego de obtenidos los resultados, se procedió a presentar los análisis en tablas, a fin de responder pertinentemente a nuestros objetivos planteados.

\section{RESULTADOS}

Tabla 1

Conglomerado de datos demográficos: edades, género, años de servicios y estatus en la investigación sobre la satisfacción laboral y el compromiso organizacional en empleados de una universidad privada de Lima, 2011.

\begin{tabular}{ccc}
\hline Edades & N. ${ }^{\circ}$ & $\%$ \\
\hline De 20 a 30 años & 14 & 11.6 \\
De 31 a 40 años & 27 & 22.3 \\
De 41 a 50 años & 32 & 26.4 \\
De 51 a 60 años & 36 & 29.9 \\
De 61 a 70 años & 12 & 9.9 \\
\hline Género & N. ${ }^{\circ}$ & $\%$ \\
\hline Masculino & 72 & 59.5 \\
Femenino & 49 & 40.5 \\
\hline Años de servicios & N. ${ }^{\circ}$ & 2.5 \\
\hline De 1 a 4 años & 3 & 42.1 \\
De 5 a 10 años & 51 & 14.0 \\
De 11 a 15 años & 17 & 19.8 \\
De 16 a 20 años & 24 & 12.4 \\
De 21 a 25 años & 15 & 7.4 \\
De 26 a 30 años & 9 & 1.7 \\
De 31 a más & 2 & \\
\hline
\end{tabular}




\begin{tabular}{ccc}
\hline Estatus & N. ${ }^{\circ}$ & $\%$ \\
\hline Operario & 60 & 49.6 \\
Administrativo & 49 & 40.4 \\
Académico & 12 & 10.0 \\
Total & 121 & 100.0 \\
\hline
\end{tabular}

De acuerdo a la tabla 1, la cantidad total de evaluados fueron 121 personas. Se debe resaltar el $29.9 \%$ como el rango de mayor frecuencia comprendido entre las edades de 51 a 60 años y el $26.4 \%$ cuyas edades se encuentran entre los 41 y 50 años, ambos rangos nos muestran que gran parte de los empleados de esta universidad privada están atravesando la etapa de adultos tardíos, siendo contrastado con los adultos jóvenes que representan solo el $11.6 \%$ de la muestra total.

Del mismo modo, esta tabla nos presenta el género de los participantes siendo el $59.5 \%$ varones mientras que el $40.5 \%$ damas; y en relación a los años de servicios; el rango mayoritario (42.1\%) está comprendido entre los 5 a 10 años de labores, representando a una población laboralmente novicia; en comparación al $1.7 \%$ que representan a los trabajadores con más de 30 años de servicio activo a la institución.
Asimismo en esta tabla se hace referencia al estatus de los empleados. El nivel operario (49.6); son todos aquellos trabajadores cuyas labores se basan en brindar sus servicios como ayudantes y mano de obra, especialmente de manera práctica en los centros de aplicación (Imprenta y Productos Unión) y la Gerencia de Servicios de la institución. El nivel administrativo, con un $40.4 \%$, hace referencia a todos aquellos trabajadores que forman parte del área administrativa de la institución, para cuyas labores se requiere de determinados estudios y/o preparación académica. Como ejemplo tenemos a la Gerencia General, Rectorado, Recursos Humanos, Gerencia Financiera y los diferentes departamentos administrativosfinancieros de la organización y con un $10 \%$ de la muestra total, el nivel académico se encuentra conformado por todos aquellos empleados que se dedican a la enseñanza universitaria en las diferentes facultades y escuelas profesionales que conforman esta casa superior de estudios.

Tabla 2

Resultado global de la satisfacción laboral en empleados de una universidad privada de Lima, 2011.

\begin{tabular}{ccc}
\hline Nivel de Satisfacción Laboral & N. $^{\circ}$ & $\%$ \\
\hline ... a 92 - Altamente Insatisfechos & 14 & 11.6 \\
93 a 111 - Insatisfechos & 48 & 39.7 \\
112 a 148 - Medianamente satisfechos & 59 & 48.7 \\
149 a 167 - Satisfechos & 0 & .0 \\
168 a más - Altamente Satisfechos & 0 & .0 \\
Total & 121 & 100.0 \\
\hline
\end{tabular}

Los datos de la tabla 2 muestran los resultados globales de la satisfacción laboral en los empleados de la institución. El rango mayoritario se ubica en la categoría de medianamente satisfechos a un $48.7 \%$, lo cual indica que los empleados tienen una satisfacción neutra, en otras palabras, trabajan solo por el ingreso económico que perciben y, por lo tanto, son fácilmente influenciables hacia conductas de riesgo para la institución (Palma, 1999). Es muy importante resaltar que ninguna persona de la muestra se considera satisfecha ni altamente satisfecha con su actividad laboral, sino por el contrario hay un índice elevado de insatisfacción de 51.6\% (39.7\% insatisfechos y 11.6\% altamente insatisfechos), esto es muy peligroso para la institución ya que puede resultar en conductas laborales muy disfuncionales (Palma 1999). 
Tabla 3

Resultado conglomerado de las dimensiones: condiciones laborales/materiales, beneficios laborales/remunerativos, políticas administrativas, relaciones sociales, desarrollo personal, desempeño de tareas y relación con la autoridad de la satisfacción laboral en empleados de una universidad privada de Lima, 2011.

\begin{tabular}{|c|c|c|}
\hline Condiciones Laborales/Materiales & $\mathrm{N} .^{\circ}$ & $\%$ \\
\hline ... a 10 - altamente insatisfechos & 15 & 12.4 \\
\hline 11 a 14 - insatisfechos & 28 & 23.1 \\
\hline 15 a 19 - regulares & 46 & 38.0 \\
\hline 20 a 22 - satisfechos & 21 & 17.4 \\
\hline 23 a más - altamente satisfechos & 11 & 9.1 \\
\hline Beneficios Laborales/Remunerativos & $\mathrm{N} .^{\circ}$ & $\%$ \\
\hline ... a 6 - altamente insatisfechos & 12 & 9.9 \\
\hline 7 a 8 - insatisfechos & 8 & 6.6 \\
\hline 9 A 14 - regulares & 76 & 62.8 \\
\hline 15 a 17 - satisfechos & 23 & 19.0 \\
\hline 18 a más - altamente satisfechos & 2 & 1.7 \\
\hline Políticas Administrativas & $\mathrm{N} .^{\circ}$ & $\%$ \\
\hline ... a 10 - altamente insatisfechos & 3 & 2.5 \\
\hline 11 a 14 - insatisfechos & 26 & 21.5 \\
\hline 15 a 19 - regulares & 66 & 54.5 \\
\hline 20 a 22 - satisfechos & 20 & 16.5 \\
\hline 23 a más - altamente satisfechos & 6 & 5.0 \\
\hline Relaciones Sociales & $\mathrm{N} .^{\circ}$ & $\%$ \\
\hline ... a 7 - Altamente Insatisfechos & 1 & 0.8 \\
\hline 8 a 11 - insatisfechos & 4 & 3.3 \\
\hline 12 a 16 - regulares & 61 & 50.4 \\
\hline 17 a 18 - satisfechos & 36 & 29.8 \\
\hline 19 a más - altamente satisfechos & 19 & 15.7 \\
\hline Desarrollo Personal & $\mathrm{N} .^{\circ}$ & $\%$ \\
\hline ... a 13 - altamente insatisfechos & 6 & 5.0 \\
\hline 14 a 18 - insatisfechos & 3 & 2.5 \\
\hline 19 a 25 - regulares & 67 & 55.4 \\
\hline 26 a 28 - satisfechos & 29 & 24.0 \\
\hline 29 a más - altamente satisfechos & 16 & 13.2 \\
\hline Desempeño de Tareas & $\mathrm{N} .^{\circ}$ & $\%$ \\
\hline ... a 13 - altamente insatisfechos & 3 & 2.5 \\
\hline 14 a 18 - insatisfechos & 5 & 4.1 \\
\hline 19 a 25 - regulares & 60 & 49.6 \\
\hline 26 a 28 - satisfechos & 31 & 25.6 \\
\hline 29 a más - altamente satisfechos & 22 & 18.2 \\
\hline
\end{tabular}




\begin{tabular}{ccc}
\hline Relación con la Autoridad & N. ${ }^{\circ}$ & $\%$ \\
\hline ... a 13 - altamente insatisfechos & 3 & 2.5 \\
14 a 18 - insatisfechos & 13 & 10.7 \\
19 a 25 - regulares & 70 & 57.9 \\
26 a 28 - satisfechos & 27 & 22.3 \\
29 a más - altamente satisfechos & 8 & 6.6 \\
Total & 121 & 100.0 \\
\hline
\end{tabular}

Los resultados de la tabla 3 muestran los puntajes de las 7 dimensiones de la satisfacción laboral. Los puntajes más sobresalientes por cada dimensión son: (I) Condiciones laborales / materiales; el 38.0\%, se ubica en la categoría regular o medianamente satisfechos, lo que indica que la institución cumple de manera básica o parcial sus necesidades de materiales e infraestructura; (II) Beneficios laborales y/o remunerativos, el 62.8\%, se ubica en la categoría regular o medianamente satisfechos, lo que indica que la institución cumple de manera básica con sus expectativas económicas y sus beneficios laborales; (III) Políticas administrativas establecidas por la institución, el $54.5 \%$ de la muestra se ubica en la categoría regular o medianamente satisfechos, lo que indica una aceptación básica de las normas o lineamientos que la institución propone; (IV) Relaciones sociales, el rango mayor de la muestra se ubica en la categoría regular o medianamente satisfechos con un $50.4 \%$, lo que indica que las relaciones sociales que se mantienen son regularmente estables y cordiales; (V) Desarrollo personal brindado, un porcentaje elevado de $55.5 \%$ se ubica en la categoría regular o medianamente satisfechos, lo que indica que los empleados consideran aceptable, en promedio, las oportunidades de desarrollo personal que la institución les brinda; (VI) Desempeño de tareas, el $49.6 \%$ se ubica en la categoría regular, lo cual indica que los empleados se sienten medianamente satisfechos con las tareas cotidianas que le son encomendadas a realizar, resaltando también al $43.8 \%$ que se encuentran satisfechos $(25.6 \%$ satisfechos y $18.2 \%$ altamente satisfechos) y; (VII) Relación con la autoridad, el rango mayor de la muestra se ubica en la categoría regular o medianamente satisfechos con un $57.9 \%$, lo que indica que la relación que mantienen los empleados con sus autoridades son regularmente estables y cordiales.

Tabla 4

Resultado global del compromiso organizacional en empleados de una universidad privada de Lima, 2011.

\begin{tabular}{ccc}
\hline Compromiso Organizacional & N. ${ }^{\circ}$ & $\%$ \\
\hline ... a 42 - sin compromiso & 0 & 0 \\
43 a 63 - con compromiso leve & 10 & 8.3 \\
64 a 84 - comprometidos & 66 & 54.5 \\
85 a más - con compromiso alto & 45 & 37.2 \\
Total & 121 & 100.0 \\
\hline
\end{tabular}

La tabla 4 nos muestra el resultado general obtenido del Compromiso Organizacional. Vemos que el $54.5 \%$ de empleados comprometidos junto al $37.2 \%$ con compromiso alto suman un total de 91.7\% de la muestra general con un buen nivel de compromiso, lo cual demuestra que los empleados se sienten identificados con su centro laboral y buscan su desarrollo como organización. Es interesante notar que solo un $8.3 \%$ presenta un compromiso leve y que no existe personas dentro de la muestra que no se sientan comprometidos con la institución. 
Tabla 5

Resultado conglomerado de las dimensiones: Participación, identificación, pertenencia y lealtad del Compromiso Organizacional en empleados de una universidad privada de Lima, 2011.

\begin{tabular}{|c|c|c|}
\hline Participación & N. ${ }^{\circ}$ & $\%$ \\
\hline ... a 12 - No participativos & 4 & 3.3 \\
\hline 13 a 18 - Levemente participativos & 31 & 25.6 \\
\hline 19 a 24 - Participativos & 81 & 66.9 \\
\hline 25 a más - Altamente participativos & 5 & 4.1 \\
\hline Identificación & N. ${ }^{\circ}$ & $\%$ \\
\hline ... a 12 - No identificados & 1 & .8 \\
\hline 13 a 18 - Levemente identificados & 6 & 5.0 \\
\hline 19 a 24 - identificados & 51 & 42.1 \\
\hline 25 a más - Altamente identificados & 63 & 52.1 \\
\hline Pertenencia & N. ${ }^{\circ}$ & $\%$ \\
\hline ... a 10 - Sin pertenencia & 2 & 1.7 \\
\hline 11 a 15 - Con pertenencia leve & 11 & 9.1 \\
\hline 16 a 20 - Con pertenencia & 49 & 40.5 \\
\hline 21 a más - Con pertenencia alta & 59 & 48.8 \\
\hline Lealtad & N. ${ }^{\circ}$ & $\%$ \\
\hline ... a 8 - Sin lealtad & 2 & 1.7 \\
\hline 9 a 12 - Con lealtad leve & 39 & 32.2 \\
\hline 13 a 15 - Leales & 47 & 38.8 \\
\hline 16 a más - Con lealtad alta & 33 & 27.3 \\
\hline Total & 121 & 100.0 \\
\hline
\end{tabular}

La tabla 5 muestra los puntajes de las 4 dimensiones del compromiso organizacional. Los puntajes más sobresalientes por cada dimensión son: (I) Participación, el 66.9\% se encuentra en la categoría participativos que, junto al $4.1 \%$ de los altamente participativos, hacen un total de $71.0 \%$; esto significa que el empleado percibe adecuadamente que, la organización le permite involucrarse en las decisiones que debe hacerse como institución; (II) Identificación, el $42.1 \%$ se encuentra en la categoría identificados, sumado al $52.1 \%$ de los altamente identificados hacen un total de $94.2 \%$; esto significa que el empleado tiene un elevado deseo de estar involucrado con la institución en todo su accionar y desarrollo; (III) Pertenencia, el $89.3 \%$ siente que pertenece a la institución (40.5\% con pertenencia y $48.8 \%$ con pertenencia alta) lo que significa que se sienten a gusto con la organización y tienen el deseo de trabajar en la misma ante cualquier situación que pueda ocurrir; y (IV) Lealtad, el 38.8\% se encuentran en la categoría de leales, los cuales junto al $27.3 \%$ de los que tienen lealtad alta hacen un total de $66.1 \%$; esto significa que el empleado tiene un elevado deseo de defender a la organización ante las críticas externas, confiando pacientemente y con optimismo en que la administración de la institución hará lo que le corresponde. 
Tabla 6

Resultado de la correlación existente entre la satisfacción laboral y el compromiso organizacional en empleados de una universidad privada de Lima, 2011

\begin{tabular}{cc}
\hline Correlaciones & Satisfacción Laboral TOTAL \\
\hline CO Participación & $0,530^{* *}$ \\
CO Identificación & $0,577^{* *}$ \\
CO Pertenencia & $0,577^{* *}$ \\
CO Lealtad & $0,468^{* *}$ \\
Compromiso organizacional TOTAL & $0,732^{* *}$ \\
\hline
\end{tabular}

**. La correlación es significativa al nivel 0,01 (bilateral).

En la tabla 6 se encuentran contenidos los resultados de la correlación existente entre la satisfacción laboral y el compromiso organizacional. Vemos que el coeficiente de correlación de Pearson para ambas variables es de 0.732 , lo cual indica que existe una correlación positiva al $95 \%$ de confiabilidad. Este valor nos da a entender que nuestra variable compromiso organizacional tenderá a aumentar, si se incrementa la satisfacción laboral.

Del mismo modo, se observa que todas las dimensiones se mantienen estables y también poseen un alto índice de correlación, confirmando el resultado obtenido de la correlación entre los totales de las variables.

\section{DISCUSIÓN}

El presente estudio analiza la relación entre la satisfacción laboral y el compromiso organizacional. Esta evaluación expone la realidad actual que es vivenciada dentro de una universidad privada.

El primer asunto a tratar será sobre la Satisfacción Laboral. Los resultados indican que los empleados de esta institución poseen $48.7 \%$ de puntaje máximo global en la escala de Satisfacción Laboral, por lo que se encuentran en el rango de medianamente satisfechos. Estas personas, según Palma (1999), tienen una actitud neutra hacia su propio trabajo; lo cual significa que son empleados que se desempeñan normalmente sin brindar esfuerzo extra o iniciativa a la actividad que realizan, haciendo solo lo necesario para cumplir los requerimientos del día o de la jornada laboral.

De otro lado, es preocupante el alto nivel de insatisfacción laboral que existe en la institución: el $51.3 \%$ se encuentran insatisfechos (39.7\% insatisfechos y $11.6 \%$ altamente insatisfechos); siendo altamente crítico, también que en la población estudiada no se encuentren personas satisfechas ni altamente satisfechas, las cuales son representadas por un temible $0 \%$. Debido a esta insatisfacción generalizada los empleados realizan conductas o acciones contraproducentes a la institución, como por ejemplo: disminuyen su desempeño laboral, disminuyen su motivación, tienen altos índices de absentismo y rotación, entran en conflictos con sus pares y superiores, no acatan órdenes y reglamentos, poseen una actitud negativa y desvalorizada de su labor y de la institución y son propensos a tener accidentes laborales (Aamodt 2010).

Palma (1999) da a conocer que la Satisfacción Laboral está conformada por siete dimensiones. En esta universidad privada hay algunas dimensiones que sobresalen y otras que se encuentran muy por debajo de la media. En relación a las sobresalientes, vemos que los empleados tienen un adecuado grado de complacencia en relación al incentivo económico (Beneficios Laborales y/o Remunerativos 20.7\%); un alto nivel de satisfacción en la interrelación con otros miembros de la organización con quienes comparten sus actividades laborales cotidianas (Relaciones Sociales 45.5\%); consideran que tienen la oportunidad de realizar actividades significativas para su autorrealización (Desarrollo Personal 37.2\%); tienen una adecuada apreciación de sus tareas cotidianas (Desempeño de Tareas $43.8 \%$ ) y valoran positivamente las relaciones con sus jefes y supervisores (Relación con la Autoridad $28.9 \%$ ). Estos puntajes son alentadores y necesitan ser reforzados para aumentar la satisfacción existente en estas dimensiones.

Asimismo, se aprecia que dos de las dimensiones estudiadas tienen predominancia hacia la insatisfacción. Los empleados se consideran insatisfechos con los elementos materiales y de infraestructura donde desenvuelven su labor cotidiana 
y no consideran que esta sea facilitadora de la labor que realizan (Condiciones Laborales y Materiales $35.5 \%$ ); y de la misma manera tienen cierto grado de desacuerdo frente a los lineamientos o normas institucionales dirigidas a regular su relación laboral y que está asociada directamente con ellos. Debido a esto, las dos dimensiones mencionadas, deberían ser un punto crucial de estudio para el planteamiento de estrategias de intervención y de esta manera mejorar la Satisfacción Laboral de los empleados; ya que si se desea prosperar como institución se debe mantener satisfechas a las personas que hacen posible que esta universidad privada continúe con su actividad, porque si se encuentren satisfechos, habrán muchos cambios y mejoras en las mismas personas y, de esta manera, su desempeño laboral se duplicará y mejorarán los estándares ya alcanzados hasta ahora, logrando así un mayor reconocimiento institucional ante la sociedad.

Otro tema importante que ha sido analizado en la presente investigación, es el Compromiso Organizacional. Los resultados revelan que, al contrario de lo que sucede con la Satisfacción Laboral, los empleados sí poseen Compromiso Organizacional, siendo el $91.2 \%$ quienes se encuentran comprometidos con la institución (54.5\% comprometidos y $37.2 \%$ con compromiso Alto); lo cual según Steers y Porter (1991), significa que los empleados tienen una adecuada identificación e involucramiento con la organización. Aquí se puede aplicar el comentario de Vance (2006), quien dice que “...los empleados que están comprometidos con sus trabajos y comprometidos hacia sus organizaciones dan a estas compañías unas ventajas competitivas muy cruciales..." de esta manera podemos avistar uno de los factores del por qué esta universidad privada es una universidad emprendedora, a pesar del déficit que tiene en brindar Satisfacción Laboral a sus empleados.

Por otro lado, el constructo Compromiso Organizacional está dividido en cuatro dimensiones (ya mencionadas); siendo los puntajes obtenidos muy favorables y alentadores. Estos resultados nos muestran que los empleados de esta universidad privada son personas que sienten que se les está permitido involucrarse en las decisiones que debe tomar la institución (Participativos 66.9\%); asimismo, desean fuertemente estar involucrados con la universidad en todo su accionar y desarrollo (Altamente Identificados $52.1 \%$ ); también se sienten muy a gusto con la organización y tienen el deseo de trabajar en la misma ante cualquier situación que acontezca (Pertenencia Alta $48.8 \%$ ) y por último, sienten el deseo de defender a su institución ante las críticas externas (leales 38.8\% y lealtad alta $27.3 \%$ ).

Por último, debemos considerar que la correlación encontrada en este estudio entre la Satisfacción Laboral y el Compromiso Organizacional es positiva. Esto nos indica que a mayor Satisfacción Laboral de los empleados también se incrementa Compromiso Organizacional también se elevará. Es importante resaltar que, si los administradores de esta universidad privada hacen el esfuerzo por aumentar la Satisfacción Laboral de sus empleados, se tendría un porcentaje mucho más alto de Compromiso Organizacional, el cual, si por el momento es alto, el resultado final sería alto en demasía y por ende muy gratificante.

\section{CONCLUSIONES}

- La satisfacción laboral en los empleados de una universidad privada de Lima en el año 2011 se encuentra dentro del rango de medianamente satisfechos; siendo este grupo determinado por el 48.7\% del Puntaje Global (PG) obtenido en la Escala de SL-SPC; sin embargo, y contrastando el porcentaje anterior, la insatisfacción laboral es el valor mayoritario y predominante de la muestra, con un $51.3 \%$ del PG dentro de la misma escala; Se debe resaltar que no existe persona, dentro de la muestra evaluada, que se encuentre satisfecho o altamente satisfecho con su actividad laboral en esta institución $(0 \%)$.

- De acuerdo a la estimación obtenida de la Escala del Grado de Compromiso de los Empleados Universitarios ASD, el $91.2 \%$ de los empleados se encuentran comprometidos con esta institución a la que pertenecen.

- Existe correlación significativa $(\mathrm{r}=.732$, $\mathrm{p}=.00$ ) entre las variables satisfacción laboral y compromiso organizacional en los empleados de una universidad privada de Lima, 2011.

- Existe correlación significativa entre la satisfacción laboral y la participación $(\mathrm{r}=.530, \mathrm{p}=.00)$ de los empleados de una universidad privada de Lima, 2011.

- Existe correlación significativa entre la satisfacción laboral y la identificación $(r=.577, \mathrm{p}=.00)$ de los empleados de una universidad privada de Lima, 2011.

- Existe correlación significativa entre la satisfacción laboral y la pertenencia $(\mathrm{r}=.577, \mathrm{p}=.00)$ de los empleados de una universidad privada de Lima, 2011.

- Existe correlación significativa entre la satisfacción laboral y la lealtad $(r=.468, \mathrm{p}=.00)$ de los empleados de una universidad privada de Lima, 2011. 


\section{REFERENCIAS BIBLIOGRÁFICAS}

Aamodt, M. (2010). Industrial/Organizational Psychology. California: Cengage Learning.

Batemen, L y Strasser, P. (1984). Organizational commitment: Antecedents and Outcomes in Public Organizations. Public Productivity and Management Review, 29, 256-277.

Bisen, V. (2010). Industrial Psychology. Delhi, India: New Age International (P) Ltd. Publishers.

Blau, P. M. (1977). Inequality and Heterogeneity. New York: Free Press.

Brief, A. (1998). A Critical Overview of Research on Organizational Attitudes. London: Sage.

Brown, B. (2003). Employees' Organizational Commitment and Their Perception of Supervisors' Relations-Oriented and Task-Oriented Leadership Behaviors. Virginia Polytechnic Institute Research Magazine, 50, 1 - 115.

Celep, C. (1999). Teachers' Level of Organizational commitment. Journal of Applied Psychology, 25, 25-96.

Côté, S., \& Heslin, P. (2003). Job Satisfaction \& Organizational Commitment. Personnel Psychology, I, 25-30.

Gellatlly, G. (1995). Configurations of Organizational Effectiveness and Efficiency. Academy of Management Journal, 36, 1345-1361.

Grajales, T. (2000). Escala de satisfacción en el trabajo entre empleados universitarios ASD. Memorias del CIE Número 1, Vol.2. 110-120.

Hellriegel, D., \& Slocum, J. (2011). Organizational Behavior (13 Ed.). Mason, $\mathrm{OH}$ : Cengage Learning.

Hernández, R., Fernández, C., \& Baptista, L. (2006). Metodología de la Investigación (4 ed.). México: McGraw-Hill.

Iaff, M. T. y Muchinsky, P. M. (1985). Job Satisfaction and Job Performance: A Meta - analysis. Psychological Bulletin, 97, 251-273.

Koopman, P. (1994). Transactional Versus Transformational Leadership. Congress of Applied Psychology, Madrid, Spain.

Kroehnert, G. (2000). Basic training for trainers (3rd ed.). New York: McGraw-Hill.
Liou, K. T. (1990). Non-work Factors and Job Satisfaction Revisited. Human Relations, 43, 7786.

Loli, A., \& Cuba, E. (2007). Autoestima y compromiso organizacional en trabajadores de una universidad pública de provincias. REVISTA IIPSI, 10, 103-108.

Loli, A. (2006). Compromiso organizacional de los trabajadores de una universidad pública de Lima y su relación con algunas variables demográficas. Revista de Investigación en Psicología UNMSM, 8, 150-185.

Mayo, E. (1924-1933). The Reasons that Affect the Productivity in Hawthrone Plant, Chicago. An Long-Term study from Harvard Business School.

McFarlane, L., \& Martin, H. (1989). Job Satisfaction and Organizational Commitment in Relation to Work Performance and Turnover Intentions. Human Relations, 42, 625-638.

Meliá, J., \&Peiró, J. (1989). La medida de la satisfacción laboral en contextos organizacionales: El Cuestionario de Satisfacción S20/23. Psicologemas, 5, 59-74.

Meyer, J. P y Allen, L. (1991). Job Satisfaction, Organizational Commitment, Turnover intention, and Turnover: Path analyses based on meta-analytic findings. Personnel Psychology, 46(2), 259-293.

Muchinsky, P. (2006). Psychology Applied to Work (8 ed.). Belmont, CA: Thomson.

Palma, C. (1999). Elaboración y validación de una escala de satisfacción laboral SL-SPC para trabajadores de Lima Metropolitana. Lima, Perú: Universidad Nacional Mayor de San Marcos.

Porter, L. W. (1974). Managerial Attitudes and Performance. Homewood, IL: Dorsey.

Psychological, A. (2009). Publication Manual of the American Psychological Association (6th ed.). Washington, DC: APA.

Reichers, K. (1985). Antecedents and Consequences of Organizational Commitment. Educational and Psychological Measurement.

Roberts, K. H., Hulin, C. L., \& Rousseau, D. M. (1978). Developing an Interdisciplinary Science of Organizations. San Francisco: Jossey-Bass. 
Rogelberg, S. (2004). Handbook of Research Methods in Industrial and Organizational Psychology. USA: Blackwell Publishing Ltd.

Rojas, M. (2002). Manual de redacción científica. Lima, Perú: UNMSM.

Runyon, R., \& Harber, A. (1980). Estadística para las ciencias sociales. México: Editorial Addison.

Steers, R. M., y Porter, L. W. (1991). Motivation and Work Behavior (4th ed.). New York: McGraw-Hill.

Tejeda, K. (2007). Factores predictores de la satisfacción laboral en los docentes de la Universidad Adventista Dominicana y del Colegio Adventista Dominicano en el 2006. Revista de Investigación Universidad de Montemorelos, 12, 78-95.

Üstüner, M. (2009). Teachers' Organizational Commitment Scale: A Validity and Reliability Study. Inonu University Journal of the Faculty of Education, 10, 1-17.

Van Dyne, L. (1993). Complex Interdependence in Task-performing Groups. Journal of Applied Psychology, 78, 61-72.

Vance, R. J. (2006). Statistical Control of Halo Error in Performance Ratings. Journal of Applied Psychology, 65, 501-6. 